\title{
Natural infection of the sand fly Phlebotomus kazeruni by Trypanosoma species in Pakistan
}

\author{
Hirotomo Kato ${ }^{1 *}$, Hiroshi Uezato ${ }^{2}$, Hiroshi Sato ${ }^{3}$, Abdul M Bhutto ${ }^{4}$, Farooq R Soomro ${ }^{5}$, Javed H Baloch', \\ Hiroyuki Iwata', Yoshihisa Hashiguchi ${ }^{6}$
}

\begin{abstract}
The natural infection of phlebotomine sand flies by Leishmania parasites was surveyed in a desert area of Pakistan where cutaneous leishmaniasis is endemic. Out of 220 female sand flies dissected, one sand fly, Phlebotomus kazeruni, was positive for flagellates in the hindgut. Analyses of cytochrome $b$ (cyt b), glycosomal glyceraldehyde phosphate dehydrogenase ( $g$ GAPDH) and small subunit ribosomal RNA (SSU rRNA) gene sequences identified the parasite as a Trypanosoma species of probably a reptile or amphibian. This is the first report of phlebotomine sand flies naturally infected with a Trypanosoma species in Pakistan. The possible infection of sand flies with Trypanosoma species should be taken into consideration in epidemiological studies of vector species in areas where leishmaniasis is endemic.
\end{abstract}

\section{Findings}

Phlebotomine sand flies are blood-sucking insects belonging to the family Psychodidae in the order Diptera [1]. The identification of sand fly species is epidemiologically very important because less than 10 percent of over 800 species described are responsible for the transmission of human pathogens such as flagellate protozoa of the genus Leishmania [1-3]. Some sand fly species are reported to transmit non-pathogenic flagellates of Endotrypanum species, originally identified as intraerythrocytic parasites of sloths in the New World [4]. In addition, some Trypanosoma species of mammals, lizards, snakes and toads are transmitted by phlebotomine sand flies [5-11]. Since the flagellated forms of these parasites in the insect gut are morphologically similar to those of Leishmania, careful differentiation is needed for the epidemiological study of the vectors responsible for circulating Leishmania species.

In this study, the natural infection of sand flies by Leishmania was surveyed in a desert area of Pakistan where cutaneous leishmaniasis is endemic. Sand flies were captured at Sono Khan $\left(26^{\circ} 52^{\prime} \mathrm{N}, 68^{\circ} 03^{\prime} \mathrm{E}\right)$, Sindh Province on the $28-29^{\text {th }}$ of June 2004 . Shannon traps with a single compartment $(1.0 \times 1.0 \times 1.3$ meters in

\footnotetext{
* Correspondence: katoh@yamaguchi-u.ac.jp

'Department of Veterinary Hygiene, Faculty of Agriculture, Yamaguchi University, 1677-1 Yoshida, Yamaguchi 753-8515, Japan
}

width, length and height, respectively) were used for the collection. The captured sand flies were dissected and the species identified based on the morphology of their spermathecae. These flies were also examined for Leishmania in the gut microscopically at $\times 400$ magnification. The flagellates detected were inoculated into Difco blood agar (USMARU) biphasic medium containing 20\% defibrinated rabbit blood, and later co-cultured with Spodoptera frugiperda Sf 9 insect cells in Grace's medium supplemented with $10 \%$ fetal calf serum. The rest of the flagellates in the gut of the sand fly were fixed in absolute ethanol for molecular biological analyses. Genomic DNA was extracted from the ethanol-fixed specimen, and the cytochrome $b(c y t \mathrm{~b})$ gene was amplified from the flagellate with primers prepared for the leishmanial cyt b gene (L.cyt-S: GGTGTAGGTTTTAGTYTAGG and L.cyt-R: CTACAATAAACAAATCATAATATRCAATT) [12]. The glycosomal glyceraldehyde phosphate dehydrogenase $(g G A P D H)$ and small subunit ribosomal RNA (SSU rRNA) genes were also amplified from the parasite using gGAPDH (G3: TTYGCCGYATYGGYCGCATGG and G5: ACMAGRTCCACCACRCGGTG) and SSU rRNAspecific primers (TRY927F: GAAACAAGAAACACGGGAG and TRY927R: CTACTGGGCAGCTTGGA) designed for trypanosomatids [13,14]. The PCR products were cloned into the pGEM-T Easy Vector (Promega, Madison, WI), and the sequences of the inserts of the plasmids were determined by the dideoxy chain 
A

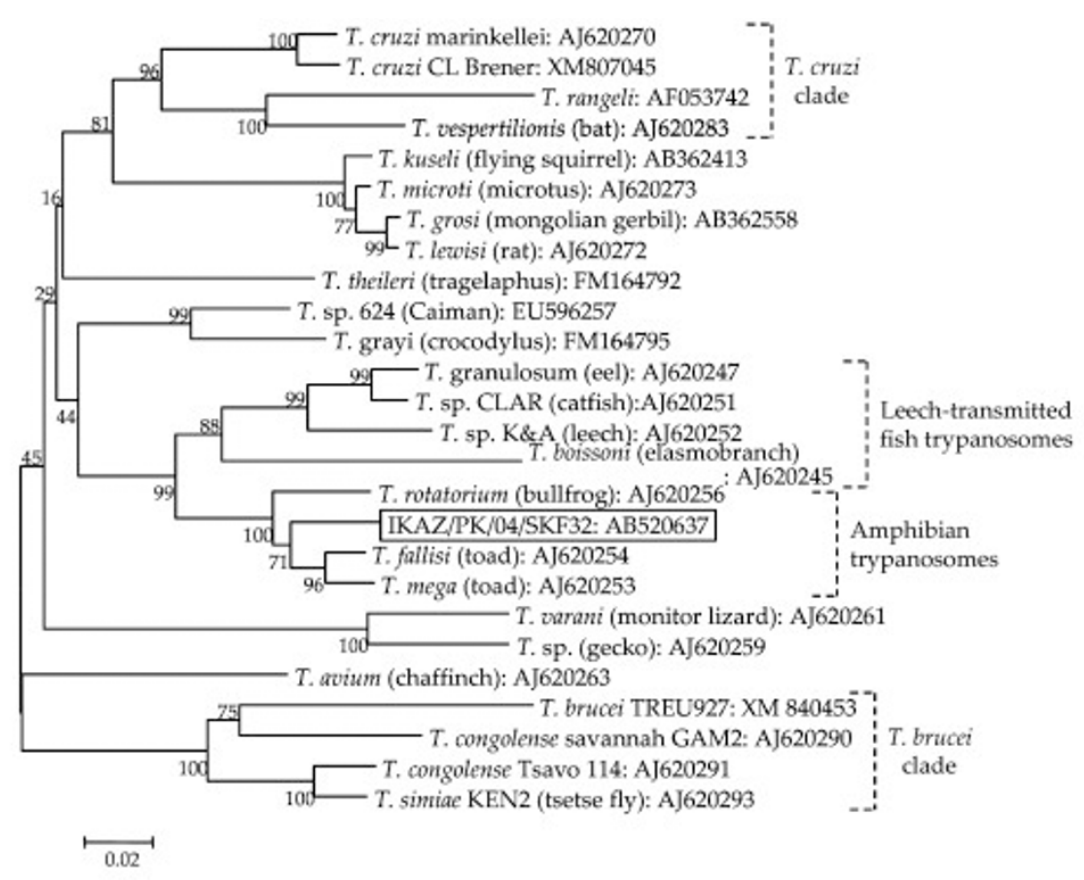

B

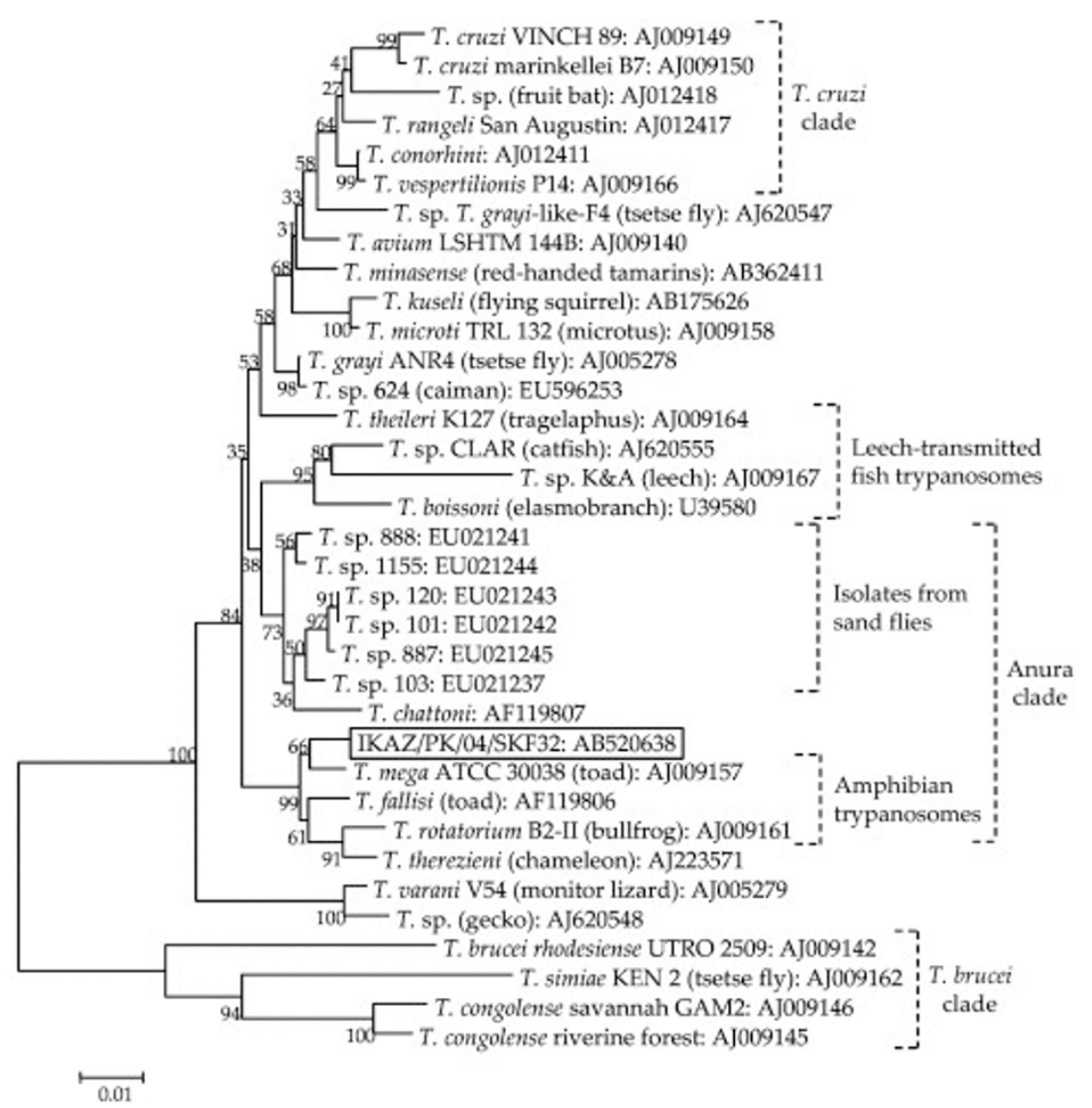

Figure 1 Phylogenetic tree of $g$ GAPDH (A) and SSU rRNA (B) gene sequences among species. The gGAPDH and SSU rRNA genes were amplified from isolates of flagellates from P. kazeruni (IKAZ/PK/04/SKF32), and the sequences were determined. Phylogenetic analyses of $g$ GAPDH and SSU rRNA gene sequences were performed by the neighbor-joining method together with those from 25 and 34 Trypanosoma species, respectively. The sequences from the database are represented by "the name of the species and isolate (host of the isolate): GenBank accession number". The scale bar represents 0.02 and $0.01 \%$ divergence, respectively. Bootstrap values are shown above or below branches. 
termination method using a BigDye Terminator v3.1 Cycle Sequencing Kit (Applied Biosystems, Foster City, CA). The gGAPDH and SSU rRNA gene sequences were aligned with CLUSTAL W software [15] and examined using the program MEGA (Molecular Evolutionary Genetics Analysis) version 4.0 [16]. Phylogenetic analyses were performed by the neighbor-joining (NJ) and maximum parsimony (MP) methods with the distance algorisms available in the MEGA package [16].

In this survey, 220 female sand flies were dissected for identification at the species level, and four species of the genus Phlebotomus were recognized. Among them, the two most prevalent species were identified as Phlebotomus (P.) sergenti (39 flies) and P. papatasi (27 flies). Other Phlebotomus species were $P$. nuri (10 flies) and $P$. kazeruni (1 fly), and the rest (143 flies) were identified as Sergentomyia species. The natural infection of sand flies by flagellates was detected in the hindgut of $P$. kazeruni. The cyt $\mathrm{b}$ gene sequence of the parasite was determined and analyzed with the BLASTn program. Unexpectedly, the sequence showed only $80.6-82.8 \%$ homology with those of Leishmania species and $83.6 \%$ homology with that of Trypanosoma (T.) brucei brucei, suggesting the flagellate belonged to the genus Trypanosoma. For further characterization, the $g G A P D H$ and SSU rRNA genes were amplified from the flagellate and the sequences were analyzed since these genes have been well studied in trypanosomatids $[13,14]$. The $g G A P D H$ and SSU rRNA genes from the parasite (DDBJ accession numbers: AB520637 and AB520638, respectively) had more than $90 \%$ sequence similarity with those of amphibian trypanosomes such as $T$. fallisi, $T$. $m e g a$ and $T$. rotatorium isolated from toad and frog. Phylogenetic analyses of the $g G A P D H$ and SSU rRNA genes showed that the flagellate within P. kazeruni belongs to a clade of amphibian trypanosomes that includes a chameleon trypanosome, T. therezieni, but the sequences did not completely match those from any reported species (Fig. 1A and $1 \mathrm{~B}$ ). In addition, the parasite from $P$. kazeruni located in a separate clade from Trypanosoma species isolated from Amazonian sand flies, which have closer relationships with anuran trypanosomes captured in Amazonia [10] (Fig. 1B). These results strongly suggested that the parasite is a novel or genetically uncharacterized Trypanosoma species of an amphibian or possibly reptile. The parasite was successfully isolated as a culture (code number: IKAZ/PK/04/ SKF32), which was confirmed to correspond to the original by the SSU rRNA gene analysis. The parasites markedly grew in Grace's medium in a co-culture with $S f 9$ insect cells. On glass-slide smears prepared from the liquid culture, rosettes of parasites with an oval shape and epimastigotes varying in shape and length were observed (Fig. 2). The parasites showed the features of
Trypanosoma species, demonstrating characteristics such as a flagellum, a kinetoplast and an undulating membrane (Fig. 2). The research area was a desert community, and no amphibian was observed during the field trip. However, numbers of geckoes were found on the walls of houses, including sites where the Shannon traps were set. Thus, two Japanese reptile species (Takydromus tachydromoides and Gekko japonicus) and one Japanese amphibian (Hyla japonica) were intraperitoneally injected with $5 \times 10^{6}$ parasites as a preliminary search for the vertebrate host of the parasite; however, no parasites were observed in peripheral blood 3 weeks after the inoculation. The possibility of mammalian hosts was also addressed since the frog trypanosome, $T$. rotatorium, has been reported to infect mice under experimental conditions [17]. The flagellate was inoculated intracutaneously into BALB/c mice and Mongolian gerbils (Meriones unguiculatus), but its DNA was not detected in peripheral blood and lymphoid tissues by PCR after 6 weeks. Based on the findings obtained by phylogenetic analyses and experimental infection, the parasite is presumably infectious to certain species of reptiles or amphibians in Pakistan. Further study is needed to identify the vertebrate host of the parasite species. The procedures were performed according to the recommendations of the ethics committee for animal experimentation, Faculty of Agriculture, Yamaguchi University.

In the present study, a Trypanosoma species was isolated from P. kazeruni, which is a xerophilic species distributing broadly throughout North Africa and West Asia with little-known behavior [18-20], and the parasite was genetically characterized. To date, trypanosomes in

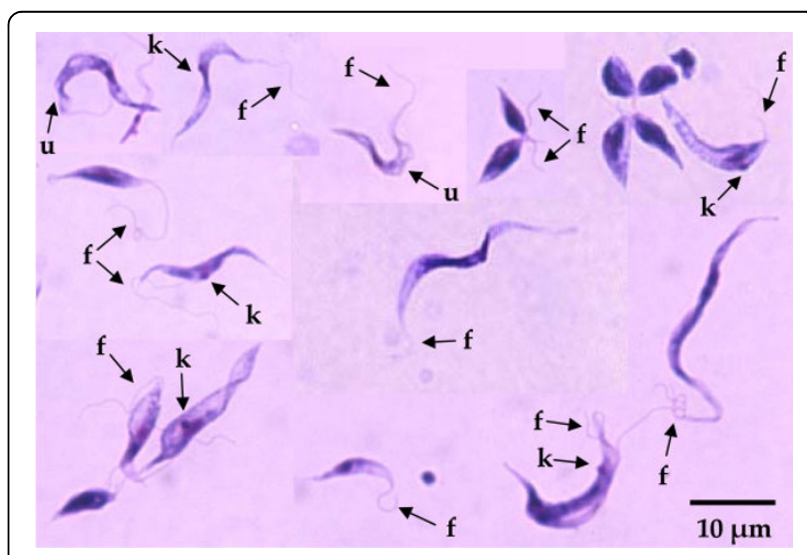

Figure 2 Trypanosoma species isolated from a phlebotomine sand fly $\boldsymbol{P}$. kazeruni. Glass-slide smears were prepared from the culture in Grace's medium co-cultivated with Sf9 insect cells and stained with Giemsa staining. Rosettes of parasites with an oval shape and epimastigotes varying in morphology were observed. k, kinetoplast; f, flagellum; u, undulating membrane. 
sand flies have been reported on the African and American continents [5-11], but this is the first record of phlebotomine sand flies naturally infected by a Trypanosoma species in Asia. Pakistan is a tropical and subtropical country located in the northwest of South Asia and has areas where leishmaniasis is highly endemic [21,22]. Although information on the endemicity and spread of cutaneous leishmaniasis is accumulating [23-26], the sand fly species responsible for the transmission is poorly understood. To date, no natural infections of sand flies by flagellates have been reported in Pakistan, and further efforts are ongoing on this matter. Thus, careful identification of the parasites is required for the epidemiological study of sand fly species responsible for the transmission of Leishmania protozoa.

\section{Acknowledgements}

We are indebted to Dr. Juma K. Kakarsulemankhel (Sandflies, Leishmaniasis \& Mosquitoes Laboratory, Department of Zoology, Faculty of Scinece, University of Balochistan, Quetta, Balochistan, Pakistan), Hamed A. Chandio and Nuzhat Seema Bhatti (The Leprosy Unit, Chandka Medical College Hospital and the Executive District Office-Health, Larkana, Sindh, Pakistan) for their support during the field phase of the present study. This study was financially supported by the Ministry of Education, Culture and Sports, Science and Technology (MEXT) of Japan (Grant Nos. 14256004, 18256004 and 18780230)

\section{Author details \\ 'Department of Veterinary Hygiene, Faculty of Agriculture, Yamaguchi University, 1677-1 Yoshida, Yamaguchi 753-8515, Japan. ${ }^{2}$ Department of Dermatology, Faculty of Medicine, University of the Ryukyus, 207 Uehara, Nishihara-cho, Okinawa 903-0215, Japan. ${ }^{3}$ Department of Veterinary Parasitology, Faculty of Agriculture, Yamaguchi University, 1677-1 Yoshida, Yamaguchi 753-8515, Japan. ${ }^{4}$ Department of Dermatology, Chandka Medical College, Doctors Colony, Bunglow No 14, VIP Road, Larkana, Sindh, Pakistan. ${ }^{5}$ Incharge Leprosy Centre, Larkana, Sindh, Pakistan. ${ }^{6}$ Department of Parasitology, Kochi Medical School, Kochi University, Oko-cho Kohasu, Nankoku-shi, Kochi 783-8505, Japan.}

\section{Authors' contributions}

HK was fully involved in all phases of the study, including field research, sample collection, laboratory work, and writing the manuscript. HU and HS were involved in the molecular works. AMB, FRS and JHB organized and conducted field research. $\mathrm{HU}$ and $\mathrm{YH}$ conducted field research and sample collection. $\mathrm{HK}, \mathrm{HI}$ and $\mathrm{YH}$ drafted the manuscript. $\mathrm{YH}$ supervised the overall conduct of the study. All authors read and approved the final manuscript.

\section{Competing interests}

The authors declare that they have no competing interests.

Received: 8 January 2010 Accepted: 25 February 2010

Published: 25 February 2010

\section{References}

1. Munstermann LE: Phlebotomine sand flies, the Psychodidae. Biology of Disease Vectors Elsevier, San Diego CA, Second 2004, 141-151.

2. Desjeux P: Leishmaniasis. Public health aspects and control. Clin Dermatol 1996, 14:417-423.

3. Bates PA: Leishmania sand fly interaction: progress and challenges. Curr Opin Microbiol 2008, 11:340-344.

4. Shaw JJ: A possible vector of Endotrypanum schaudinni of the sloth Choloepus hoffmanni, in Panama. Nature 1964, 201:417-418.

5. Anderson JR, Ayala SC: Trypanosome transmitted by Phlebotomus : first report from the Americas. Science 1968, 161:1023-1025.
6. Ayala SC: Trypanosomes in wild California sandflies, and extrinsic stages of Trypanosoma bufophlebotomi. J Protozool 1971, 18:433-436.

7. Ayala SC, McKay JG: Trypanosoma gerrhonoti n. sp., and extrinsic development of lizard trypanosomes in California sandflies. J Protozool 1971, 18:430-433.

8. Naiff RD, Barrett TV, Freitas RA: Isolation of Trypanosoma freitasi (Kinetoplastida: Trypanosomatidae) from Psychodopygus claustrei (Diptera: Psychodidae). Mem Inst Oswaldo Cruz 1989, 84:273-275.

9. Viola LB, Campaner M, Takata CS, Ferreira RC, Rodrigues AC, Freitas RA, Duarte MR, Grego KF, Barrett TV, Camargo EP, Teixeira MM: Phylogeny of snake trypanosomes inferred by SSU rDNA sequences, their possible transmission by phlebotomines, and taxonomic appraisal by molecular, cross-infection and morphological analysis. Parasitology 2008, 135:595-605.

10. Ferreira RC, De Souza AA, Freitas RA, Campaner M, Takata CS, Barrett TV, Shaw JJ, Teixeira MM: A phylogenetic lineage of closely related trypanosomes (Trypanosomatidae, Kinetoplastida) of anurans and sand flies (Psychodidae, Diptera) sharing the same ecotopes in brazilian amazonia. J Eukaryot Microbiol 2008, 55:427-435.

11. Lemos M, Morais DH, Carvalho VT, D'Agosto M: First record of Trypanosoma chattoni in Brazil and occurrence of other Trypanosoma species in Brazilian frogs (Anura, Leptodactylidae). J Parasitol 2008, 94:148-151.

12. Kato H, Cáceres AG, Gomez EA, Mimori T, Uezato H, Marco JD, Barroso PA, Iwata $H$, Hashiguchi $Y$ : Molecular mass screening to incriminate sand fly vectors of Andean-type cutaneous leishmaniasis in Ecuador and Peru. Am J Trop Med Hyg 2008, 79:719-721.

13. Noyes HA, Stevens JR, Teixeira M, Phelan J, Holz P: A nested PCR for the ssrRNA gene detects Trypanosoma binneyi in the platypus and Trypanosoma sp. in wombats and kangaroos in Australia. Int J Parasitol 1999, 29:331-339.

14. Hamilton PB, Stevens JR, Gaunt MW, Gidley J, Gibson WC: Trypanosomes are monophyletic: evidence from genes for glyceraldehyde phosphate dehydrogenase and small subunit ribosomal RNA. Int J Parasitol 2004, 34:1393-1404.

15. Thompson JD, Higgins DG, Gibson TJ: CLUSTAL W: improving the sensitivity of progressive multiple sequence alignment through sequence weighting, position-specific gap penalties and weight matrix choice. Nucleic Acids Res 1994, 22:4673-4680

16. Tamura K, Dudley J, Nei M, Kumar S: MEGA4: Molecular Evolutionary Genetics Analysis (MEGA) software version 4.0. Mol Biol Evol 2007 24:1596-1599.

17. Hysek J, Zizka Z: Transmission of Trypanosoma rotatorium from frogs to white mice. Nature 1976, 260:608-609.

18. Kamhawi S, Abdel-Hafez SK, Molyneux DH: The behaviour and dispersal of sandflies in Ras el Naqb, south Jordan with particular emphasis on Phlebotomus kazeruni. Parassitologia 1991, 33:307-314.

19. Hanafi HA, Kanour WW Jr, Beavers GM, Tetreault GE: Colonization and bionomics of the sandfly Phlebotomus kazeruni from Sinai, Egypt. Med Vet Entomol 1999, 13:295-298.

20. Toprak S, Ozer N: Sand fly species of Sanliurfa province in Turkey. Med Vet Entomol 2005, 19:107-110.

21. Khan SJ, Muneeb S: Cutaneous leishmaniasis in Pakistan. Dermatol Online J 2005, 11:4

22. Katakura K: Molecular epidemiology of leishmaniasis in Asia (focus on cutaneous infections). Curr Opin Infect Dis 2009, 22:126-130.

23. Marco JD, Bhutto AM, Soomro FR, Baloch JH, Barroso PA, Kato H, Uezato H, Katakura K, Korenaga M, Nonaka S, Hashiguchi Y: Multilocus enzyme electrophoresis and cytochrome $b$ gene sequencing-based identification of Leishmania isolates from different foci of cutaneous leishmaniasis in Pakistan. Am J Trop Med Hyg 2006, 75:261-266.

24. Noyes HA, Reyburn H, Bailey JW, Smith D: A nested-PCR-based schizodeme method for identifying Leishmania kinetoplast minicircle classes directly from clinical samples and its application to the study of the epidemiology of Leishmania tropica in Pakistan. J Clin Microbiol 1998, 36:2877-2881.

25. Bhutto AM, Soomro RA, Nonaka S, Hashiguchi Y: Detection of new endemic areas of cutaneous leishmaniasis in Pakistan: a 6-year study. Int J Dermatol 2003, 42:543-548.

26. Myint CK, Asato Y, Yamamoto $Y$, Kato H, Bhutto AM, Soomro FR, Memon MZ, Matsumoto J, Marco JD, Oshiro M, Katakura K, Hashiguchi Y, 
Uezato H: Polymorphisms of cytochrome $b$ gene in Leishmania parasites and their relation to types of cutaneous leishmaniasis lesions in Pakistan. J Dermatol 2008, 35:76-85.

doi:10.1186/1756-3305-3-10

Cite this article as: Kato et al:: Natural infection of the sand fly

Phlebotomus kazeruni by Trypanosoma species in Pakistan. Parasites \&

Vectors 2010 3:10.

Submit your next manuscript to BioMed Central and take full advantage of:

- Convenient online submission

- Thorough peer review

- No space constraints or color figure charges

- Immediate publication on acceptance

- Inclusion in PubMed, CAS, Scopus and Google Scholar

- Research which is freely available for redistribution

Submit your manuscript at www.biomedcentral.com/submit

() BioMed Central 